\title{
All-ceramic tooth-supported single crowns have acceptable 5-year survival rates
}

\section{Abstracted from \\ Wang X, Fan D, Swain MV, Zhao K.}

A systematic review of all-ceramic crowns: clinical fracture rates in relation to restored tooth type. Int J Prosthodont. 2012; 25: 441-450.

Address for correspondence: Dr Ke Zhao, Department of Prosthodontics, Guanghua School of Stomatology, Hospital of Stomatology, Sun Yat-sen University, No. 56 Lingyuan West Road, 510055 Guangzhou, China. E-mail: zhaoke@mail.sysu.edu.cn

\section{Question: What are the clinical fracture rates of all-ceramic crowns in different tooth types?}

Data sources The databases Medline/PubMed, EMBASE, the Cochrane Library, Chinese Biomedical Literature Database and the China National Knowledge Infrastructure were searched. Additional hand searches were conducted in the journals.

Study selection Randomised controlled trials, prospective cohort studies and retrospective studies with follow-up of 36 months or longer were included.

Data extraction and synthesis Data were extracted independently in duplicate. The annual core and veneer fracture rates of various tooth types were estimated and compared using Poisson regression. Results 37 studies were included; two RCTs, 25 prospective cohorts and 10 retrospective studies. Based on the calculated results, allceramic crowns had an acceptable overall five-year fracture rate of $4.4 \%$ irrespective of the materials used. Five-year fracture rates were significantly higher for molar crowns (8.1\%) compared to premolar crowns (3.0\%), and the difference between anterior (3.0\%) and posterior crowns (5.4\%) also achieved significance. Core fracture rates had a five-year incidence of $2.5 \%$, and a significantly higher core fracture rate was found in the posterior region (3.9\%). The overall five-year incidence of veneer fracture was 3.0\%, and no clear difference was found between restored tooth types, with incidences of $2.0 \%, 2.5 \%, 1.0 \%$, and $3.0 \%$ for incisor, canine, premolar, and molar crowns, respectively. Conclusions Within the limitations of this study's protocol, the current evidence suggests that dental ceramic materials demonstrated acceptable five-year core and veneer fracture incidences when used for tooth-supported single crowns in both anterior and posterior segments. Higher fracture tendency for posterior crowns was the trend for all-ceramic crowns, while molar crowns showed a significantly higher fracture rate than premolar crowns. Randomised controlled trials with large sample sizes be undertaken to obtain more definitive results.

\section{Commentary}

This review investigated the clinical fracture incidence of tooth-supported all-ceramic crowns. The review provided data on the annual core and veneer fracture rates of various tooth types. Authors searched English and Chinese databases from 1990 to 2011. They also hand searched a number of relevant dental journals. However, hand searching, unlike electronic searches, was limited to the years 2000 to 2011. Furthermore, the grey literature was not included in the searches for this review.
The review included clinical randomised controlled trials, prospective cohort and retrospective studies with mean follow-up of at least 36 months. Case reports, in-vitro and animal studies were excluded. The data were analysed using poisson regression. Authors introduced a variable, namely log (exposure), in the model to account for the different sample sizes and follow up times. It is not clear, however, how other confounding factors, including the study design, were accounted for. It is assumed that inclusion of prospective and retrospective non-controlled studies may lead to an overestimation of the actual survival rate. Furthermore, there was no provision for assessing the quality of studies, eg risk of bias. The authors indicated that the high fracture rate of some glass-ceramic materials, which are rarely recommended at present, may lead to an overestimation of the results. This assumption was supported by the results from data analysis. The annual fracture incidence for different tooth types considerably decreased following exclusion of glass-ceramic materials.

The authors considered evaluating the fracture incidence of various ceramic materials used for different tooth types separately. However, due to the small number of studies in each group this was unlikely to achieve significant results. Although the authors pointed out that in this study the role of tooth type of the fractured crowns was emphasised rather than the ceramic system, they could assess the fracture incidence of different ceramic materials with acceptable combined sample sizes (eg Procera AllCeram, In-Ceram Alumina, IPS Empress, IPS Empress 2 and Dicor), irrespective of tooth type. This would help estimate the fracture rate for different ceramic systems, which may be different from the overall results reported for ceramic materials. For example, whilst this review reported an overall five-year fracture rate of $8.1 \%$ for different ceramic systems, excluding glass-ceramic materials, another review indicated a failure rate of $10.2 \%$ for Procera AllCeram molar crowns at five or more years. ${ }^{1}$

\section{Practice point}

- All-ceramic tooth-supported single crowns demonstrated acceptable five-year survival rate in both anterior and posterior segments

- Molar all-ceramic single crowns showed a significantly higher fracture rate than other teeth.

Mojtaba Dorri School of Oral and Dental Sciences, Bristol Dental School, Bristol, UK.

1. Kassem AS, Atta O, El-Mowafy O. Survival rates of porcelain molar crowns-an update. Int J Prosthodont. 2010; 23: 60-62.

Evidence-Based Dentistry (2013) 14, 47. doi:10.1038/sj.ebd.6400932 